However, despite exceedingly rare in most countries spectinomycin resistance is annually identified in Russia, and accordingly the level of this resistance is crucial to monitor. Continuous, quality assured and quality controlled gonococcal AMR surveillance in Russia is crucial. RU-GASP now also works under WHO protocols.

\section{P1-S1.43 ANTIMICROBIAL SUSCEPTIBILITY AND MOLECULAR EPIDEMIOLOGICAL CHARACTERISTICS OF NEISSERIA GONORRHOEAE IN 2009-2010 IN BELARUS}

doi:10.1136/sextrans-2011-050108.43

${ }^{1} \mathrm{~S}$ Glazkova, ${ }^{2} \mathrm{M}$ Unemo, ${ }^{2} \mathrm{D}$ Golparian, ${ }^{1} \mathrm{~L}$ Titov, ${ }^{3} \mathrm{~N}$ Pankratova, ${ }^{4} \mathrm{~N}$ Suhabokava, ${ }^{4}$ I Shimanskaya, ${ }^{2} \mathrm{G}$ Ignatyev, ${ }^{5} \mathrm{M}$ Domeika. ${ }^{1}$ The Republican Research and Practical Center for Epidemiology and Microbiology, Belarus, ${ }^{2}$ Swedish Reference Laboratory for Pathogenic Neisseria, Orebro, Sweden; ${ }^{3}$ Mogilev Regional Dermato-Venerological Dispensary, Belarus; ${ }^{4}$ Minsk City Dermato-Venerological Dispensary, Belarus; ${ }^{5}$ Uppsala University, Sweden

Background Antimicrobial resistance (AMR) in Neisseria gonorrhoeae is a global concern, and gonorrhoea may become untreatable. However, AMR data from Eastern Europe are scarce beyond Russia, and in Belarus no AMR data or other characteristics of gonococci have been published in over 20 years. The aim was to describe the $\mathrm{AMR}$ and molecular epidemiological characteristics of gonococci circulating in 2009 and 2010 in Belarus.

Methods $N$ gonorrhoeae isolates cultured in $2009(\mathrm{n}=80)$ and 2010 $(n=78)$ in the two different cities Minsk $(n=45)$ and Mogilev $(n=113)$ were examined using Etest for nine antimicrobials and nitrocefin solution for $\beta$-lactamase production. Where available, breakpoints for susceptibility and resistance according to The European Committee on Antimicrobial Susceptibility Testing (EUCAST; http://www.eucast.org) were used. Moreover, screening for penA mosaic alleles, full-length porB gene sequencing, and $N$ gonorrhoeae multiantigen sequence typing (NG-MAST) were performed.

Results The levels of resistance in 2009-2010 to antimicrobials evidently used in the gonorrhoea treatment in Belarus wereceftriaxone $0 \%$, spectinomycin $0 \%$, azithromycin $14.4 \%$, tetracycline $30.9 \%$, ciprofloxacin $34.6 \%$, and erythromycin $59.2 \%$ (only tested in 2009). The levels of resistance to other antimicrobials of international interest but not used in Belarus were-cefixime $0 \%$, gentamicin 1.3\%, and penicillin G 9.9\% (only tested in 2009). Extraordinarily, no $\beta$-lactamase producing isolates were detected. The circulating $N$ gonorrhoeae population identified was very heterogeneous and contained many divergent NG-MAST STs, of which more than half have not been previously described worldwide.

Conclusions Due to the high levels of resistance to all antimicrobials previously recommended as first-line treatment, only ceftriaxone and spectinomycin can be recommended for empirical gonorrhoea treatment in Belarus. Continuous and quality assured gonococcal AMR surveillance in Eastern Europe is crucial, in Belarus this surveillance is now initiated using WHO protocols.

\section{P1-S1.44 ANTIMICROBIAL SUSCEPTIBILITY PROFILE OF NEISSERIA GONORRHOEAE ISOLATES IN THE PROVINCE OF QUÉBEC - 2010}

doi:10.1136/sextrans-2011-050108.44

B Lefebvre, A M Bourgault. Laboratoire de santé publique du Québec, Institut national de santé publique du Québec, Sainte-Anne-de-Bellevue, Canada

Background Regular monitoring of antimicrobial resistance of $N$ gonorrhoeae is necessary to detect the trend in antimicrobial resistance, increases in minimum inhibitory concentrations (MICs) and to assist in the development of treatment guidelines.
Methods In 2010, all N gonorrhoeae strains isolated in clinical laboratories throughout the province were submitted to the Laboratoire de santé publique du Québec where their susceptibility profile to azithromycin, cefixime, ceftriaxone, ciprofloxacin and spectinomycin was determined by the agar dilution method.

Results A total of 831 strains isolated from 607 males and 224 females were tested. The strains were recovered from (data available for 819 strains) the following specimens-urethra, 432; cervix, 191; anus, 100; throat, 90; eye, 4; and synovial fluid, 2. All strains were susceptible to cefixime, ceftriaxone and spectinomycin, 270 (32.5\%) were resistant to ciprofloxacin and $11(1.3 \%)$ were resistant to azithromycin (MIC $\geq 2 \mathrm{mg} / \mathrm{l}$ ). The azithromycin resistant isolates were retrieved from 11 males aged 16-55 years (mean, 30) from the Montreal area (urethra, 8; throat, 3). The azithromycin MIC distribution was as follows $-4 \mathrm{mg} / \mathrm{l}(\mathrm{n}=1), 8 \mathrm{mg} / \mathrm{l}(\mathrm{n}=6), 16 \mathrm{mg} / \mathrm{l}$ $(n=4)$. No highly resistant organism (MIC $>128 \mathrm{mg} / \mathrm{l}$ ) was identified. Among the azithromycin resistant isolates, one was resistant to ciprofloxacin but all were susceptible to cefixime, ceftriaxone and spectinomycin. In 2010, 68 (8.2\%) isolates exhibited decreased susceptibility to cefixime (MIC $=0.125 \mathrm{mg} / \mathrm{l}[\mathrm{n}=62]$ and $0.25 \mathrm{mg} / \mathrm{l}$ $[n=6])$. Of these 68 isolates, all were susceptible to azithromycin and ceftriaxone but all were resistant to ciprofloxacin. Only one isolate showed decreased susceptibility to ceftriaxone (MIC $=0.125$ $\mathrm{mg} / \mathrm{l}$ - - this isolate also showed decreased susceptibility to cefixime. Conclusions As observed elsewhere, $N$ gonorrhoeae strains are showing a worrisome drift in susceptibility to cefixime, a first line treatment for gonococcal infections associated with resistance to ciprofloxacin. Furthermore, resistance to azithromycin, a second line treatment option, is emerging. Sustained collaborative laboratory surveillance programs are mandatory for the design of public health interventions to prevent and control gonococcal infections.

\section{P1-S1.45 NEISSERIA GONORRHOEAE ANTIMICROBIAL SUSCEPTIBILITY IN LATIN AMERICA AND THE CARIBBEAN (2000-2009) - A CONTRIBUTION TO THE TREATMENT GUIDELINES REVISION}

doi:10.1136/sextrans-2011-050108.45

S Starnino, M Liao, M Ruben, A Storey, J A R Dillon, GASP-LAC Network*. Vaccine and Infectious Disease Organization, University of Saskatchewan, Saskatoon, Canada

Background Active surveillance of the antimicrobial susceptibility (AS) of Neisseria gonorrhoeae $(\mathrm{Ng})$ isolates was carried out by the Gonococcal Antimicrobial Surveillance Program (GASP) in Latin America and the Caribbean (LAC) during the 1990s. A retrospective analysis of surveillance activities undertaken during the 2000s was conducted to describe trends in $\mathrm{Ng}$ AS and to re-initiate the GASP network.

Methods $\mathrm{Ng}$ isolates were collected and tested for AS to penicillin (Pen), tetracycline (Tet), ciprofloxacin (Cip), ceftriaxone (Cef), azithromycin (Azi) and spectinomycin (Spe) in 11 countries between 2000 and 2009. Agar dilution, disc diffusion and Etest methods were used as described by CLSI and previously established in the GASP-LAC network. Trends in AS were retrospectively analysed in each country and aggregated at the GASP-LAC Coordinating Centre.

Results 6 countries collected data over the entire study period and five countries tested for AS sporadically. In total, $9026 \mathrm{Ng}$ isolates were tested. Cip resistance first appeared in 2000 with 2\%, (19/784) and resistant per cent increased to $30 \%$ (297/975) in 2009. Cip resistance was observed in 10 countries. Azi resistance per cent increased from $6 \%(41 / 646)$ in 2000 to $27 \%$ (224/842) in 2009; one country reported no resistance. Pen resistance per cents ranged between 29\% (299/1035) in 2000 and 33\% (256/772) in 2006. Tet resistance percents declined from 61\% (633/1041) in 2000 to $35 \%$ 
(323/931) in 2009. The majority of $\mathrm{Ng}$ isolates were susceptible to Cef and Spe. However, 7/110 isolates showed decreased susceptibility to Cef in one country in 2007 and 2/25 isolates showed intermediate susceptible to Spe in another country in 2009.

Conclusions Cip for primary treatment of uncomplicated gonococcal infections is currently recommended in 10 of the 11 countries, 1 country recommended Cef in 2007. The emergence and spread of resistance to Cip and Azi, indicate that current treatment guidelines be reviewed using respective national antimicrobial surveillance data. The emergence of a few strains with reduced susceptibility to third-generation cephalosporins indicates that on-going GASP surveillance is warranted.

*Authors contributed equally and are listed in an alphabetical order of country names. P Galarza, I. Pagano, M. E. Trigoso, A Schwartz Benzaken, V M Pinto, A Maldonado Ballesteros, 0 M Sanabria Cruz, A Llop, E Aguilar Jarrin, N Aguayo, J L Portilla Carbajal, G Borthagaray, A Acevedo, D Payares.

\section{Epidemiology poster session 1: STI trends: Syphilis \\ P1-S1.46 SYPHILIS TRENDS IN ZAMBIA - A 14-YEAR OBSERVATION}

doi:10.1136/sextrans-2011-050108.46

M Makasa, K Fylkesnes, I Sandoy. University of Bergen, Bergen, Norway

Background Syphilis and other STIs are prevalent in sub-Saharan Africa, perhaps due to lack of effective curative services. Estimates among pregnant women and in the general population in Zambia range from $6 \%$ to $18 \%$, with maternal syphilis contributing to a high number of mid-trimester abortions, still births, prematurity, and morbidity and mortality of the child. Existing evidence suggests that control of syphilis and other ulcerative and non-ulcerative STIs remains one of the key strategies for HIV control. There are no recent studies from Zambia comparing syphilis prevalence with HIV trends.

Objectives To investigate trends in syphilis prevalence using Antenatal Clinic (ANC) sentinel surveillance data, and to validate the findings with population-based data.

Methods The analyses are based on ANC data from 1994 to 2008 from 22 sites. Estimates are compared with data from Zambia Demographic and Health Surveys (ZDHS) 2001/2002 and 2007. Analyses were restricted to women aged $15-49$ years and 15-24 years. ORs with 95\% CIs were used to test whether the prevalence trends were significant.

Results The overall syphilis prevalence dropped during the period 1994-2008 among both urban and rural women aged 15-49 years from $9.8 \%$ to $2.8 \%$ and $7.5 \%$ to $3.2 \%$, respectively. Provincial variation was observed with a tendency towards a reduction. A sharper decline in syphilis prevalence was observed among women with more years in school than among those with fewer schooling years. A declining trend was also seen among young primi gravid women aged 15-19 years. A comparison of ZDHS 2001/2002 and 2007 findings also showed an overall reduction in syphilis prevalence in the general population.

Conclusions There was an overall reduction in syphilis prevalence and this decline was sharper than the decline in HIV during the same period. In part this could be attributed to the revamping of STI services in the country. Reduction in both HIV and syphilis prevalence could also suggest a reduction in the disease incidence and is likely to partially reflect positive sexual behaviour changes. Provincial variations were striking and need to be further studied to better guide specific STI prevention and control programmes in different geographical settings.

\section{P1-S1.47 MEASURING AND PRIORITISING CONGENITAL SYPHILIS CONTROL IN GUANGDONG CHINA - A MARKOV MODEL TO INFORM POLICY IMPLEMENTATION}

doi:10.1136/sextrans-2011-050108.47

${ }^{1} \mathrm{~J}$ Tucker, ${ }^{2} \mathrm{~N}$ Tan, ${ }^{3} \mathrm{C}$ Rydzak, ${ }^{4} \mathrm{~L} \mathrm{G}$ Yang, ${ }^{5} \mathrm{R}$ Peeling, ${ }^{6} \mathrm{X}$ S Chen. ${ }^{1}$ Harvard Medical School, USA; ${ }^{2}$ Harvard Institute for Global Health, Cambridge, USA; ${ }^{3}$ Beth Israel Deaconess Medical Center, USA; ${ }^{4}$ Guangdong Provincial STI Control Center, China; ${ }^{5}$ London School of Hygiene and Tropical Medicine, UK; ${ }^{6}$ National STD Control Center, China

Background Syphilis is a major public health problem in many regions of China, with increases in congenital syphilis (CS) cases causing particular concern. Precisely determining the extent of CS burden has been challenging given incomplete prenatal screening coverage and limited CS diagnostic capacity. As a result, both net under and over-reporting are plausible. In addition, there are a range of policies that could be expanded as part of the new National Syphilis Control Plan. This paper uses a Markov model to estimate the number of CS cases and other adverse outcomes and prioritise control measures in Guangdong Province, China.

Methods We developed a Markov model that incorporated agestratified fertility rates, female adult syphilis reported cases, and empirical syphilis transmission rates to estimate the number of adverse pregnancy outcomes associated with prenatal syphilis infection on a yearly basis from 2005 to 2009. Guangdong Province was the focus of this analysis given access to high quality demographic and public health data required for model inputs. Adverse outcomes examined included CS, stillbirth, neonatal death, and low birth weight. Sensitivity analyses were performed to identify variables most influential for achieving $\mathrm{WHO}$ and Chinese program benchmarks.

Results Our model estimates 264 CS cases per 100000 live births in Guangdong during 2009, substantially greater than the 129 reported CS cases per 100000 live births. This is consistent with a net 49\% under-reporting of CS cases in 2009. Higher syphilis prevalence among women in their peak childbearing years $(20-30$ years old) is closely related to greater numbers of CS cases see Abstract P1-S1.47 Figure 1. Expanding prenatal screening coverage was the single most important mechanism for preventing CS cases; increasing prenatal screening from $50 \%$ to $100 \%$ coverage would be associated with 98 CS cases averted per 100000 live births. Alternatively, a comprehensive syphilis control strategy including 100\% prenatal screening, $100 \%$ treatment completion, and $100 \%$ early screening in pregnancy would be associated with 212 CS cases averted per 100000 live births.

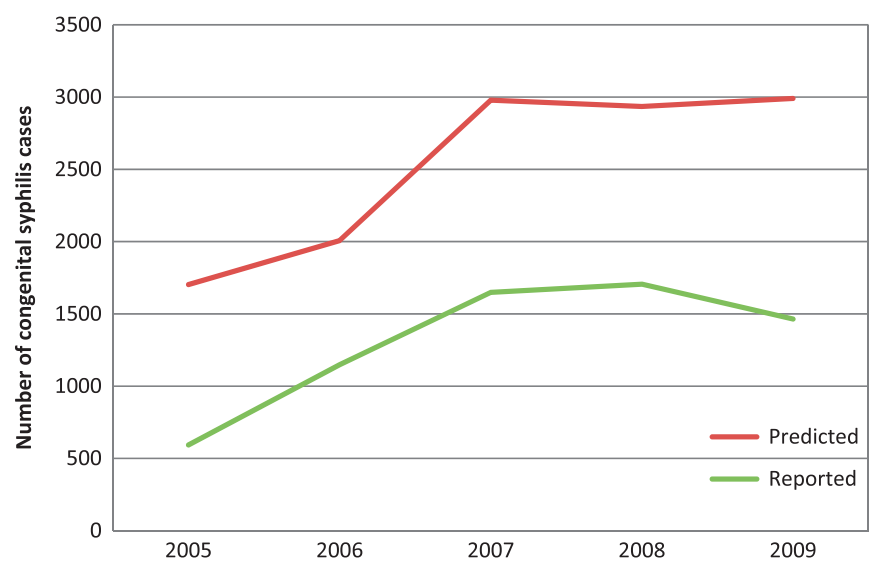

Abstract P1-S1.47 Figure 1 Predicted and reported CS cases in Guangdong Province, China, from 2005 to 2009. 


\section{LETTER}

Unusual increase in reported HIV/ AIDS cases among older persons in western Hunan province, China

An unusual increase in HIV/AIDS cases among older people was reported to the Hunan Centers for Disease Control between 2005 and 2007. Cases originated in four rural, western districts of this inland province of China. Given the historical concern for outbreaks of HIV in rural areas due to blood donation, ${ }^{1}$ these cases prompted closer examination to understand the reasons for their appearance and to take measures to prevent further spread.

Eighty cases met our investigation criterion of 50 years or older and underwent a structured interview. The median age was 65 years (range $51-82$ ); $42 \%$ were female. Most were ethnic minorities, $76 \%$ Tujia and $9 \%$ Miao, with low education. Nearly all had been married; 43\% were widowed. Most (54\%) spent time away from their spouse (median >5 years); $10 \%$ were currently sexually active with a spouse; few ever used condoms with their spouse.

Investigation of the possible modes of HIV acquisition suggests most infections among men were from female sex workers (83\% paid for sex, two-thirds in the last 5 years), and among women through infected husbands. One case had a history of selling blood, most recently in 1981. Eight received a blood transfusion, three before 1976 and five after 1984. All denied drug use; all men denied male-male sex; all women denied extramarital sex. Among men buying sex, $97 \%$ never used condoms.

Two-thirds had never heard of HIV prior to their diagnosis. Few (14\%) knew HIV could be transmitted sexually, through blood $(11 \%)$ or from mother to child $(4 \%)$. Of the men reporting commercial sex contact, $82 \%$ had no knowledge that condoms could prevent HIV. Most cases $(86 \%)$ were detected incidentally during the course of treatment for other diseases or because their spouse was HIV-positive. By interview, $78 \%$ indicated their spouse had tested for HIV, of whom $69 \%$ were reported to be positive.

Our investigation highlights that basic information on HIV/AIDS is not reaching all parts of China, and may especially lag among rural and older people. Discussion of sex with older people has been taboo in China, presenting special challenges in finding effective ways to reach them. As treatment extends survival, the cohort of persons living with HIV will also age. The movement of people between urban and rural areas, an ageing population and the shift of the HIV/AIDS epidemic to sexual transmission ${ }^{2}$ are three trends in China that may now have a dangerous intersection.

Acknowledgements We thank Dr Willi McFarland for his editorial assistance. Xi Chen, ${ }^{1}$ Jun Zheng, ${ }^{1}$ Jian Mei He, ${ }^{1}$ BiYun Qin, ${ }^{1}$
Yifei Hu, ${ }^{2}$ Lu Wang, ${ }^{2}$ Ning Wang ${ }^{2}$

${ }^{1}$ AIDS/STI Division, Hunan Provincial CDC, Changsha, China; ${ }^{2}$ The Office of State Council AIDS Working committee(SCAWCO), National Center for HIV/AIDS

Control and Prevention/China CDC, Beijing, China

Correspondence to Dr Yifei Hu, China National Center for HIV/AIDS Control and Prevention/China CDC, No.27 Nanwei Rd, Xuanwu District, Beijing, China; huyifei@yahoo.com

Funding This work was funded by the China National Mega-project of Science Research No.

2008ZX10001-003.

Competing interests None.

Patient consent Obtained.

Ethics approval This study was approved by NCAIDS/China CDC (FWA00001501).

Contributors $\mathrm{XC}$ oversaw and coordinated the fieldwork. JZ, JMH and BYO conducted the fieldwork. $\mathrm{YH}$ completed the survey design, data analysis and drafting of the manuscript. LW and NW provided technical support during and prior to the survey and mobilised some funds to complete the survey.

Provenance and peer review Not commissioned; internally peer reviewed.

Accepted 3 August 2011

Published Online First 26 August 2011

Sex Transm Infect 2011;87:538

doi:10.1136/sextrans-2011-050228

\section{REFERENCES}

1. Wu Z, Liu Z, Detels R. HIV-1 infection in commercial plasma donors in China. Lancet 1995;346:61-2.

2. China Ministry of Health, UNAIDS, and World health Organization (WHO). 2009 Estimates for the HIVIAIDS Epidemic in China. Beijing, China: China Ministry of Health, 2010. http://www.unaids.org.cn/ download/2009\%20China\%20Estimation\%20Report-En. pdf (accessed 1 May 2011).

\section{CORRECTIONS}

doi:10.1136/sextrans-2011-050102.38corr1

RamaKrishnan A. Symposium 9: Applications of program science in the field of STI: S9.3 The programme science of scale: the Avahan experience. Sex Transm Infect 2011;87:A10. doi:10.1136/ sextrans-2011-050102.38.

The author list for this abstract should read: RamaKrishnan A, Sgaier S. doi:10.1136/sextrans-2011-050108.527corr1

Patel S, Pond M. P4-S4.02: A 22-Organism Microarray Approach for Detecting Microbiological Associations with Symptomatic Urethritis in Males. Sex Trans Infect 2011;87:A316. doi:10.1136/ sextrans-2011-050108.527.

The author list for this abstract should read:

Pond $\mathrm{MJ}^{1}$, Patel $\mathrm{S}^{2}$, Hinds $\mathrm{J}^{1}$, Newton $\mathrm{R}^{3}$, Wernisch $\mathrm{L}^{3}$, Butcher $\mathrm{PD}^{1}$ and Sadiq $\mathrm{ST}^{1,2}$

1. Centre for Infection and Immunity, Division of Clinical Sciences, St George's, University of London, UK

2. Department of Genitourinary Medicine, St George's Healthcare NHS Trust, London, UK

3. MRC Biostatistics Unit, Institute of Public Health, Cambridge, UK

A pan-pathogen microarray for detection of microbiological associations with symptomatic urethritis in males.

doi:10.1136/sextrans-2011-050108.45-050108.172corr

Starnino S, Liao M, Ruben M, Storey A, Dillon JAR, GASP-LAC Network. P1-S1.45 Neisseria Gonorrhoeae Antimicrobial Susceptibility in Latin America and the Caribbean (2000-2009) - A Contribution to the Treatment Guidelines Revision. Sex Transm Infect 2011;87:A117. doi:10.1136/ sextrans-2011-050108.45.

Starnino S, Liao M, Ruben M, Storey A, Dillon JAR, GASP-LAC Network. P1S4.28 Survey Of Methodology Used For The Identification And Antimicrobial Susceptibility Testing of Neisseria Gonorrhoeae In Latin America And The Caribbean. Sex Transm Infect 2011;87:A172. doi:10.1136/sextrans-2011050108.172.

The author lists for these abstracts should read: Starnino S, GASP-LAC Network, Liao M, Ruben M, Storey A, Dillon JAR.

doi:10.1136/sextrans-2011-050109.147corr1

Campbell LA, Zidal SV, Patton D, Chochou Kuo. O4-S2.03: An Anti-Adhesive Approach to Prevention of $C$ Trachomatis Infection. Sex Trans Infect 2011;87:A86. doi:10.1136/sextrans-2011-050109.147.

The author's name Zidal SV should be correctly spelt as S Zarate Vidal. 